accumulating data now highlight an overlap between these risk loci and cellspecific enhancer elements that is maximal in CD4+ lymphocytes, followed by B lymphocytes

Objectives: Seeking insight into genetic risk mechanisms, we conducted and compared expression quantitative trait locus (eQTL) analyses of risk loci in CD4+ $T$ cells and B cells from carefully phenotyped early arthritis patients naïve to therapeutic immunomodulation.

Methods: 254 patients donated RNA and DNA from purified B and/or CD4+ T-cells within 4 hours of blood draw. Genotyping and global gene expression measurement were carried out using the Illumina Human CoreExome array and either HT12v4 or WG6v3 BeadChip arrays respectively. Variants in linkage disequilibrium (LD) with 101 confirmed non-HLA RA- SNPs $\left(r^{2}>0.8\right)$ were analysed, seeking evidence of cis- or trans- eQTLs according to whether associated probes were or were not within $4 \mathrm{MB}$ of these LD blocks.

Results: Genes subject to cis eQTL effects common to both CD4+ and Blymphocytes at RA risk loci were FADS1, FADS2, BLK, FCRL3, ORMDL3 and GSDMB. At the 8p23 BLK-FAM167A locus, we found adjacent genes subject to eQTLs whose activity differed markedly between cell types, the FAM167A effect displaying striking B-lymphocyte specificity. By contrast, cis eQTLs acting on METTL21B, IKZF3, and PADI4 were unique to CD4+ lymphocytes, the latter two of these being identified for the first time in this cell subset. No trans eQTLs approached experiment-wide significance, and linear modelling did not identify a significant influence of biological co-variates (diagnosis, systemic inflammation, age) upon eQTL effect sizes.

Conclusions: Our findings refine understanding of candidate causal genes in RA pathogenesis, providing an important platform from which downstream functional studies may be prioritised and directed towards particular cell types.

References:

[1] Okada Y, Wu D, Trynka G, Raj T, Terao C, Ikari K, et al. Genetics of rheumatoid arthritis contributes to biology and drug discovery. Nature. 2014 Feb 20; 506(7488):376-381.

Acknowledgements: Wellcome Trust, Academy of Medical Sciences, JGW Patterson Foundation, National Institute of Health Research, Pfizer. Newcastle researchers received infrastructural support via the Arthritis Research UK Centre of Excellence for the RA pathogenesis (RACE). JM and NN are funded by an MRC/Arthritis Research UK stratified medicine award (MR/K015346/1).

Disclosure of Interest: N. Thalayasingam: None declared, J. Massey: None declared, A. Anderson: None declared, N. Nair: None declared, A. Skelton: None declared, D. Lendrem: None declared, L. Reynard: None declared, H. Cordell: None declared, S. Eyre: None declared, A. Barton: None declared, J. Isaacs: None declared, A. Pratt Grant/research support from: Pfizer

DOI: 10.1136/annrheumdis-2017-eular.4028

\section{THU0004 CROSS PHENOTYPE ASSOCIATION MAPPING OF THE MHC IDENTIFIES GENETIC VARIANTS THAT DIFFERENTIATE PSORIATIC ARTHRITIS FROM PSORIASIS}

J. Bowes ${ }^{1}$, J. Ashcroft ${ }^{1}$, N. Dand ${ }^{2}$, P. Ho ${ }^{1,3}$, C.H. Smith ${ }^{4}$, J.N. Barker ${ }^{4}$ N. McHugh ${ }^{5}$, R.B. Warren ${ }^{6}$, A. Barton ${ }^{1,3}$ on behalf of BSTOP and BADBIR. ${ }^{1}$ Arthritis Research UK Centre for Genetics and Genomics, The University of Manchester, Manchester; ${ }^{2}$ Division of Genetics and Molecular Medicine, King's College London, London; ${ }^{3}$ NIHR Manchester Musculoskeletal Biomedical Research Unit, Central Manchester NHS Foundation Trust, Manchester; ${ }^{4}$ St John's Institute of Dermatology, Guys and St Thomas' Foundation Trust, London; ${ }^{5}$ Royal National Hospital for Rheumatic Diseases and Dept Pharmacy and Pharmacology, University of Bath, Bath; ${ }^{6}$ The Dermatology Centre, Salford Royal NHS Foundation Trust, Manchester, United Kingdom

Background: The identification of genetic variants that differentiate PsA from psoriasis has the potential to help us understand the underlying biological pathways that lead to the development of PsA. Associations to genetic variants within the major histocompatibility complex (MHC), in particular to HLA-C 0602 , increase risk of both PsA and psoriasis when compared to control populations. However direct comparisons of PsA to psoriasis have led to paradoxical associations where HLA-C*0602 has been reported to be protective of PsA. In addition $H L A-C^{*} 0602$ has been reported to be associated with age of onset of psoriasis. A more recent study has reported the amino acid at position 45 of the HLA-B protein as the most important factor for differentiating PsA from psoriasis. Objectives: Here we perform a cross phenotype association analysis in an attempt to identify genetic variants in the MHC that differentiate PsA from psoriasis in a large collection of PsA patients and psoriasis patients screened for the absence of PsA.

Methods: A total of 1069 patients with psoriasis and 981 patients with PsA from the UK were genotyped using either the Illumina Immunochip or the Illumina OmniExpress genotyping arrays. SNPs, amino acids and classical HLA alleles were imputed using SNP2HLA. Logistic regression was used to compare the imputed dosage of MHC markers between PsA and psoriasis. All analyses were repeated using age of psoriasis onset as an additional covariate.

Results: The most significant association when comparing PsA to psoriasis was to HLA-C*0602 $\left(\mathrm{p}=4.17 \times 10^{-15}\right)$ with a protective effect for PsA (OR $0.52, \mathrm{Cl}$ $0.44: 0.61)$. HLA- $C^{*} 0602$ was found to be significantly associated with a younger age of psoriasis onset $\left(p=1.51 \times 10^{-60}\right)$ where the median age of onset in years for carriage is 22 compared to 33 for non-carriage. We observed a difference in the median age of psoriasis onset in years between the PsA and psoriasis study subgroups (34 vs. 21), highlighting the potential for bias at markers associated with age of psoriasis onset. When controlling for the age of psoriasis onset in the analyses we observed no association of PsA to HLA-C*0602 $(p=0.07)$ and the most significant association was to the amino acid at position 97 of HLA-B $\left(p=1.54 \times 10^{-9}\right)$ where the presence of asparagine or serine residue increased risk of PsA. Asparagine at position 97 of HLA-B defines the HLA-B*2705 allele.

Conclusions: Comparing PsA to psoriasis we show HLA-C*0602 confers no effect, either risk or protective, for PsA after correction for age of psoriasis onset. The results suggest that the previously observed protective effect of HLA-C*0602 could be due to confounding due to a younger age of psoriasis onset in the psoriasis subgroup. When accounting for age of psoriasis onset, the primary association conferring risk for PsA in patients with psoriasis is to HLA-B amino-acid 97 where an asparagine residue defines the HLA-B*2705 allele. In addition, this amino acid has been reported as the largest genetic effect for ankylosing spondylitis thereby refining the genetic overlap between these two spondyloarthropathies.

Disclosure of Interest: None declared

DOI: 10.1136/annrheumdis-2017-eular.3403

\section{THU0005 ERAP POLYMORPHISMS AND ITS ASSOCIATION WITH HLA-B15 AND HLA-B27 POSITIVE SPONDYLARTHRITIS PATIENTS}

J. Londono ${ }^{1}$, A.M. Santos ${ }^{2}$, J.C. Rueda ${ }^{2}$, P. Peña ${ }^{2}$, I. Briceño ${ }^{3}$

E.-L. Saldarriaga ${ }^{2}$, J.-I. Angarita ${ }^{2}$, E. Calvo ${ }^{4}$, M. Avila ${ }^{2}$,

N. Martinez-Rodriguez ${ }^{5}$, H. Cubides ${ }^{2}$, V. Parra ${ }^{2}$, J.F. Medina ${ }^{6} .{ }^{1}$ Reumatología,

Universidad de la Sabana-Hospital Militar Central, Bogotá; ${ }^{2}$ Reumatología;

${ }^{3}$ Facultad de Medicina, Grupo Genética Humana, Universidad de la Sabana, Chia; ${ }^{4}$ Departamento de Imágenes diagnósticas, Universidad Nacional, Bogotá, Colombia; ${ }^{5}$ Departamento de Investigación de Salúd Comunitaria, Hospital Infantil de México Federico Gomez, Ciudad de México, Mexico; ${ }^{6}$ Departamento de Medicina Interna, Escuela de Medicina, Universidad de Navarra, Pamplona, Spain

Background: Since 1973, the association of HLA-B27 and spondyloarthritis $(\mathrm{SpA})$ is well known, however in Colombian population it is present in only $40 \%$ of patients and HLA-B15 is present almost in $25 \%$. A mechanism of polygenic mechanism has been proposed as an explanation for the development of SpA. Endoplasmic reticulum aminopeptidase (ERAP) genes 1 and 2 have been implicated. ERAP1 is strongly associated with HLA-B27 positive patients and ankylosing spondylitis, but not with ERAP2

Objectives: To determine the association between ERAP polymorphisms and HLA-B27 or HLA-B15 positive SpA patients

Methods: 178 patients with SpA according to ASAS criteria were included in the study. HLA typing was performed by the PCR technique using the Biorad ${ }^{\circledR}$ HLA-SSP plates. The polymorphisms were determined by the RT-PCR technique using Roche ${ }^{\circledR}$ probes for ERAP1 rs27044, rs17482078, rs10050860, and rs 30187. For ERAP2 the probes used were rs2910686, rs2248374 and rs2549782. The

Table 1. ERAP2 Haplotypes in HLA-B15 and B27 Patients

\begin{tabular}{lcccc}
\hline Haplotypes & $\begin{array}{c}\text { HLA B15 } \\
\mathrm{n}(\mathrm{AF})\end{array}$ & $\begin{array}{c}\text { HLA B27 } \\
\mathrm{n}(\mathrm{AF})\end{array}$ & $\begin{array}{c}\text { OR } \\
\text { (CI 95\%) }\end{array}$ & $p$ \\
\hline TGT & $\mathbf{0 . 2 0 1}$ & 0.078 & $2.943(1.264-6.585)$ & $0.009^{*}$ \\
TGC & 0.055 & $\mathbf{0 . 2 2 7}$ & $4.483(1.524-13.187)$ & $0.003^{*}$ \\
CAT & 0.021 & $\mathbf{0 . 1 1 9}$ & $9.014(1.181-68.807)$ & $0.009^{*}$ \\
CAC & 0.643 & 0.499 & $1.750(0.968-3.162)$ & 0.077 \\
CGC & 0.016 & 0.035 & $0.465(0.053-4.056)$ & 0.672 \\
CGT & 0.031 & 0.013 & $2.406(0.332-17.45)$ & 0.584 \\
TAT & 0.019 & 0.013 & $1.185(0.106-13.29)$ & 1.00 \\
TAC & 0.013 & 0.015 & $1.185(0.106-13.29)$ & 1.00 \\
\hline
\end{tabular}

ERAP: endoplasmic reticulum aminopeptidase; AF: allelic frequency; OR: odds ratio.

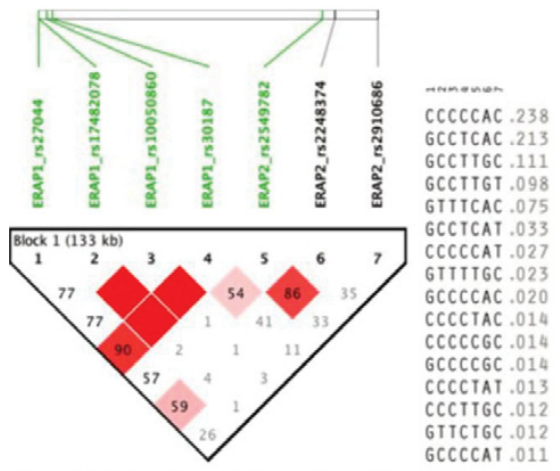

Figure 1. Linkaye disequilibrium (LD) map of the ERAP gene. The value of the LD coefficiant in each cell D'[(values close to 1.0$) \times 100]$ is shown. The colour map of the haplogroups represent the LD relationships, the bright red squares represant evidence of LD (log odds [LOD] score $<2$ the $L$ relationships, the bright red squares ropresant evidence of $L$ (log odds [LOD] score $\angle 2$
and $D-1]$, the light ral squars indicate a slight widence of $L D$ (log odds [LOD] score $\geqslant 2$ and $D<1$, and white squares mean that there is no $L D$ and independent segrogation [log odds [LOD] score $<2$ and $D<11$ 